\title{
L'accord du verbe en nombre à l'écrit chez des élèves de la fin de l'élémentaire en Ontario français: une étude de cas
}

\author{
Joël Thibeault ${ }^{1}$ et Carole Fleuret ${ }^{2}$ \\ ${ }^{1}$ Université de Regina, Canada \\ ${ }^{2}$ Université d'Ottawa, Canada \\ Article received 10 October 2015, accepté 17 March 2016 version finale 21 March 2016 \\ DOI: http://dx.doi.org/10.5565/rev/jt13.651
}

\begin{abstract}
Résumé
Cet article présente les résultats préliminaires d'une étude de cas multiples menée auprès de huit élèves scolarisés en français à la fin de l'ordre élémentaire dans le sud-ouest de l'Ontario, au Canada, là où cet idiome est langue minoritaire. En nous appuyant sur un cadre théorique hybride, composé de travaux en didactique des langues première et seconde, nous avons comme objectif la description de leurs connaissances relatives à l'accord du verbe en nombre à l'écrit. Les analyses montrent que le pluriel verbal est encore en construction chez nos participants et que les résultats varient énormément d'un élève à l'autre. Ils permettent également la mise en lumière d'une difficulté importante, le changement du radical des verbes à bases multiples lors du processus d'accord, une caractéristique qui n'est documentée que par des chercheurs qui se sont intéressés à l'apprentissage de l'écrit en français langue seconde.
\end{abstract}

Mots-clés: Accord du verbe en nombre, écriture, Ontario français, milieux minoritaires, grammaire

\begin{abstract}
This article presents the preliminary results of a multiple-case study conducted with eight students at the end of elementary school who are schooled in French in Southwest Ontario (Canada), where it is a minority language. Based upon a hybrid theoretical framework that combines the works of researchers focusing on learning French as a first and as a second language, its objective is to describe how our participants make verbs agree in number with their subjects when they write. Our analyses show that verbal plurality is still under construction for our participants and that their results greatly fluctuate from one student to the other. They also shed light on an important difficulty faced by these students, the alteration of the stem for irregular verbs, which is only documented by studies on second language French writing.
\end{abstract}

Keywords: Subject-verb agreement in number, writing, French Ontario, minority settings, grammar

\section{Resumen}

Este artículo presenta los resultados preliminares de un estudio de caso múltiple con ocho estudiantes al final de la escuela primaria cuya enseñanza es en francés en 
el sudoeste de Ontario (Canadá), donde esta lengua es minoritaria. Está basado en un marco teórico híbrido que combina los trabajos de investigadores, centrándose en el aprendizaje del francés como primer y segundo idioma, su objetivo es describir cómo los participantes hacen que los verbos concuerden en número con el sujeto al escribir. Nuestros análisis demuestran que la pluralidad verbal aún está en construcción para nuestros participantes y que sus resultados varían en gran manera de un estudiante a otro. También sacan a la luz una dificultad significativa que afrontan estos estudiantes que es la alteración del radical de los verbos irregulares, que sólo está documentada por estudios de la escritura de lengua francesa como segundo idioma.

Palabras claves: Concordancia en número entre sujeto-verbo, escritura, francés en Ontario, entorno social minoritario, gramática

\section{Problématique}

S'il y a bien une dimension de l'écrit qui est polémique et qui est source d'inquiétudes pour plusieurs scripteurs, jeunes et adultes, c'est bien l'orthographe grammaticale! Ayant fait l'objet de plusieurs travaux dans les vingt dernières années (Ouellet, Wagner et Boily, 2013; Fayol et Got, 1991), l'apprentissage de cette composante du système orthographique peut effectivement s'avérer complexe, et ce, pour plusieurs raisons. D'abord, elle impose au scripteur la prise en considération d'un ensemble de marques morphologiques qui, pour la plupart, ne sont pas associées à la chaine sonore. Ainsi, pour développer les automatismes qui réduiront le cout cognitif lié à leur mise à l'écrit, l'apprenant devra avant toute chose s'engager dans un processus réflexif, métalinguistique, et comprendre les relations qui unissent les mots dans la phrase.

Plus récemment, la recherche portant sur l'apprentissage orthographique a ouvert ses portes à des perspectives empiriques qui vont au-delà du seul paradigme épistémologique cognitiviste et qui postulent que les connaissances du scripteur en la matière peuvent être tributaires d'une myriade de facteurs complexes et diversifiés, qu'ils soient d'ordre affectif ou culturel (Boyer, 2012; Fleuret et Armand, 2012). Dans cette perspective, Totereau, Brissaud, Reilhac et Bosse (2013) ont montré que l'appréhension des composantes grammaticales de l'orthographe était particulièrement sensible socialement, en ce sens qu'elle variait selon le contexte géographique dans lequel le scripteur évolue. C'est dans cette optique que nous avons envisagé la réalisation de notre recherche doctorale, qui vise notamment à décrire les 
connaissances relatives à l'accord du verbe en nombre à l'écrit chez des élèves de la fin de l'ordre élémentaire en Ontario. Cet article présente un aperçu de nos résultats.

\section{Une note sur l'éducation de langue française en Ontario}

L'article 23 de la Charte canadienne des droits et libertés garantit une scolarité dans la langue de la minorité officielle à ceux dont l'un des parents a fréquenté un établissement scolaire appartenant à cette minorité. En Ontario, où approximativement $5 \%$ de la population se dit francophone, ce sont quelque 425 écoles qui offrent des programmes éducatifs de langue française (ministère de l'Éducation de l'Ontario, 2011). Eu égard aux élèves, certains d'entre eux ont grandi dans un foyer au sein duquel les deux parents sont francophones, alors que d'autres, de plus en plus nombreux dans la province, sont issus de l'union d'un couple exogame français-

anglais $^{1}$ ou d'une famille où le français n'est pas langue de communication. À cela s'ajoute au demeurant les élèves provenant de familles immigrantes, fort nombreux en Ontario, qui arrivent sur les bancs d'école avec un bagage linguistique qui leur est propre et qui, d'après Gérin-Lajoie et Jacquet (2008), vivent un processus de triple minorisation : ils sont immigrants, immigrants francophones où le français est minoritaire et possiblement immigrants francophones appartenant à une minorité visible.

Devant cette indéniable hétérogénéité linguistique, la recherche en sociologie de l'éducation (Duquette, 2006; Gérin-Lajoie, 2012) tend d'ailleurs à montrer que les élèves des écoles de langue française en Ontario, n'ayant donc pas toujours un contact fréquent avec le français, se construisent une identité hybride, au carrefour d'au moins deux langues-cultures. En didactique, Blain (2003) soutient également que le recours à un cadre théorique double, qui puise sa source dans les travaux de chercheurs en didactique du français langues première (L1) et seconde (L2), favorisera une compréhension fine des enjeux qui sont afférents à la scolarisation en contexte de minorisation linguistique. Ainsi les assises conceptuelles sur lesquelles repose notre étude proviennent-elles de la recherche sur l'apprentissage de l'accord verbal en nombre au sein de deux disciplines connexes, l'apprentissage du français L1 et L2, la première étant à ce jour bien plus riche que la seconde.

Bellaterra Journal of Teaching \& Learning Language \& Literature. 9.3 (Aug-Sept 2016) ISSN 2013-6196 


\section{L'accord du verbe en nombre à l'écrit en L1}

Les premiers travaux ayant porté sur l'accord du verbe en nombre ont mis au jour une série d'étapes par l'entremise desquelles il se développerait. L'étude pionnière de Totereau, Thévenin et Fayol (1997) a d'ailleurs montré que l'interprétation des marques du pluriel précède leur production et que le traitement de la flexion nominale est plus précoce que celui de son versant verbal. Quand le traitement de l'accord du verbe en nombre est amorcé, les élèves auraient d'abord tendance à commettre des erreurs de surgénéralisation en transposant aux verbes la marque du pluriel des noms (p. ex., ils *parles), jusqu'à ce qu'ils automatisent les flexions adéquates $^{2}$. Au Québec, l'étude de Lefrançois (2009), qui s'est attardée aux raisonnements métalinguistiques qu'appliquent des élèves du primaire pour marquer le pluriel graphique, montre que la conception des scripteurs de 5e année relativement au nombre grammatical demeure fragile. En ce qui a trait précisément aux formes conjuguées des verbes, Boyer (2012) argüe qu'il existe un écart important entre les taux de réussite des élèves de première secondaire. Certaines formes, comme celles des verbes avoir et être, seraient toutefois mieux réussies que d'autres, en raison de leur haute fréquence d'occurrence. La chercheure note enfin que la complexité morphosyntaxique de la phrase jouera un rôle de premier ordre dans le choix de la flexion verbale de l'élève et que, par exemple, le non-respect de l'ordre canonique sujet-prédicat-complément et la présence d'un écran entre le verbe et son donneur d'accord pourront influer sur la mise à l'écrit de la désinence.

\section{L'accord du verbe en nombre à l'écrit en L2}

Là où les travaux sur le marquage du nombre verbal menés auprès d'élèves francophones ont surtout porté sur le choix de la flexion adéquate et les processus cognitifs qui le permettent, les quelques études en L2 (Ågren, 2005, 2008; Gunnarsson, 2012), de leur côté, ont mis en lumière une caractéristique qui n'a pas été documentée par les chercheurs en L1: le marquage lexical de l'accord verbal en nombre. En effet, lors du processus d'accord, le scripteur doit non seulement s'interroger sur la désinence qui respecte les conventions linguistiques, il doit aussi, pour les verbes à bases multiples, faire usage de son répertoire lexical et sélectionner le radical approprié. 
La fréquence des formes verbales irrégulières étant élevée en français, l'élève dont le français est la L1 n'éprouvera que rarement de la difficulté à s'appuyer sur des connaissances associées à l'évocation lexicale lors du processus d'accord. Pour l'apprenant du français L2, la situation est tout autre.

Assez souvent, au début de l'apprentissage du français langue seconde, les élèves recourent à des formes non fléchies lorsqu'une telle forme est nécessaire (p. ex., ils *aller) ou ils se trompent dans le choix du radical des verbes à bases multiples (p. ex., ils *allent). Puis, en ce qui concerne particulièrement l'assignation du nombre verbal à l'écrit, les verbes irréguliers se construisant à l'aide d'une forme supplétive à la troisième personne du pluriel (être/avoir/faire/aller) commenceront à être fléchies correctement ${ }^{3}$. Suivront les verbes dont la forme infinitive se termine en -er, dont la distinction singulier-pluriel n'est pas perceptible à l'oral, et les autres verbes affichant plus d'un radical.

\section{L'accord du verbe en nombre à l'écrit en milieu francophone minoritaire}

Il devient dès lors intéressant, au regard des travaux menés en L1 et en L2, de se questionner sur l'accord du verbe en nombre à l'écrit chez des élèves qui ne sont que peu exposés au français à l'extérieur de l'enceinte scolaire. À partir des données collectées par des chercheurs auprès d'étudiants universitaires, on peut d'ores et déjà souligner que le domaine verbal représente la pierre d'achoppement des scripteurs en Ontario français (Bernier, 2013; Laflamme et Reguigui, 2003). À notre connaissance, la recherche ne nous apprend toutefois rien sur les connaissances des élèves du primaire quant à l'accord verbal en nombre à l'écrit en contexte de minorité linguistique; nous savons néanmoins que ces connaissances demeurent parcellaires chez les élèves québécois à la fin de cet ordre d'enseignement (Boyer, 2012; Lefrançois, 2009), et ce, même si le curriculum de français au Québec (ministère de l'Éducation du Québec, 2001), tout comme celui de l'Ontario (ministère de l'Éducation de l'Ontario, 2006), stipule que cette notion devrait, dans les cas simples, être apprise à ce moment de la scolarité obligatoire. 


\section{Méthodologie}

Pour décrire les connaissances d'élèves de la fin de l'ordre élémentaire sur l'accord du verbe en nombre à l'écrit en Ontario français, nous avons misé sur une recherche qualitative, laquelle se réalise sous la forme d'une étude de cas multiples (Gagnon, 2012). Dans le cadre de ce texte, nous ne présenterons que quelques données préliminaires, celles-ci provenant de l'un des nos instruments de mesure: l'activité de complètement ${ }^{4}$.

\section{Population}

Pour atteindre notre objectif de recherche, nous avons recouru à un échantillon composé de huit élèves, quatre de cinquième primaire (10-11 ans), quatre de sixième primaire (11-12 ans), tous scolarisés au sein d'une même classe à niveaux multiples dans le sud-ouest de l'Ontario. La diversité ethnolinguistique étant un trait caractérisant la population scolaire qui nous intéresse, il nous importe de mettre en exergue les langues qui sont utilisées dans le milieu familial de nos participants:

Tableau 1: langue(s) que les participants utilisent lorsqu'ils parlent à leurs parents

\begin{tabular}{lll}
\hline Élève $^{5}($ niveau scolaire) & Langue(s) parlée(s) avec la mère & Langue(s) parlée(s) avec le père \\
\hline Sabrina $\left(\mathbf{6}^{\mathbf{e}}\right)$ & Anglais, français, espagnol & Anglais \\
Pierre $\left(\mathbf{6}^{\mathbf{e}}\right)$ & Créole haïtien & Créole haïtien \\
Kate $\left(\mathbf{6}^{\mathbf{e}}\right)$ & Anglais, français & Anglais \\
Isaac $\left(\mathbf{6}^{\mathbf{e}}\right)$ & Swahili, français & Français, anglais \\
Emma $\left(\mathbf{5}^{\mathbf{e}}\right)$ & Anglais & Anglais \\
Maya $\left(\mathbf{5}^{\mathbf{e}}\right)$ & Anglais, français & Anglais \\
Ali $\left(\mathbf{5}^{\mathbf{e}}\right)$ & Arabe, français, anglais & Anglais, arabe, français \\
Alicia $\left(\mathbf{5}^{\mathbf{e}}\right)$ & Anglais, tagalog, français & Anglais \\
\hline
\end{tabular}

Les répertoires langagiers que se sont construits les élèves prenant part à cette étude sont donc marqués par une pluralité évidente. Cela étant dit, à l'exception d'Isaac, qui dit n'être que francophone, l'ensemble des participants se déclare autant francophone qu'anglophone, ce qui est un phénomène typique des écoles ontariennes de langue française (Gérin-Lajoie, 2012). De plus, depuis leur arrivée sur les bancs d'école, ils sont scolarisés en français. 


\section{Instrumentation}

Afin de rendre compte de leurs connaissances sur l'accord du verbe en nombre à l'écrit, nous avons initialement utilisé trois dispositifs méthodologiques: une activité de complètement, une production écrite et un entretien métagraphique (Jaffré, 1995), dans le cadre duquel nous nous sommes entretenus avec chacun des élèves individuellement pour qu'ils nous expliquent comment ils ont présidé aux choix de leurs graphies. Pour chacun des outils, deux passations ont eu lieu, la première en janvier 2015 et l'autre en mai 2015, pour que les portraits que nous brossons des élèves soient les plus fidèles possible. Dans cet article, comme nous l'avons mentionné précédemment, nous ne présenterons qu'un aperçu des résultats de l'activité de complètement, dont le contenu a fait l'objet d'une validation externe ${ }^{6}$ et d'une préexpérimentation auprès de sept élèves de cinquième année de la même école que nos participants ${ }^{7}$.

Plusieurs variables ont été contrôlées lors de la conception de l'instrument. D'abord, l'élaboration des contextes phrastiques s'est opérée à la suite d'une recension de la littérature sur l'influence de l'environnement morphosyntaxique sur l'accord verbal (voir notamment Cogis, 2013 en L1 et Gunnarsson, 2012 en L2). Nous avons également choisi les verbes constitutifs de nos items à partir de la typologie des verbes proposée par Ågren (2008) en L2, celle-ci en distinguant trois catégories. La première inclut les verbes en -er (p. ex., parle/parlent), le deuxième renferme les verbes dotés d'une forme supplétive à la troisième personne du pluriel (être/avoir/faire/aller) et la dernière, les autres verbes. Au total, lors de l'activité de complètement, chaque élève devait accorder 48 verbes. Un tableau présentant les contextes morphosyntaxiques retenus pour la construction des items se trouve à l'annexe A. Celui-ci indique également le nombre de verbes qui appartiennent à chacune des trois catégories identifiées par Ågren.

\section{Analyse}

L'analyse des données est divisée en deux volets, le premier portant sur l'assignation des marques morphologiques liées au nombre et l'autre focalisant sur le radical verbal. De ce fait, si l'élève a proposé la graphie $I l s$ *dirent, il a obtenu le point consacré à la marque morphologique, 
mais n'a pas obtenu celui de la base. En revanche, s'il a orthographié $I l s * t i e n n e$, nous lui avons attribué un point pour le radical, mais il n'en a reçu aucun pour la flexion. Rappelons que les résultats présentés ici sont préliminaires et que les données des entretiens métagraphiques et des productions écrites, de concert avec une analyse plus fine des données collectées lors de l'activité de complètement, nous aideront à mieux comprendre l'accord verbal en nombre chez nos participants.

\section{Quelques résultats et discussion}

La présentation des résultats s'articulera autour de deux axes: nous présenterons d'abord les scores généraux relatifs aux flexions et aux radicaux pour chacun des huit élèves lors des deux passations, et nous nous attacherons ensuite à la présentation détaillée des résultats de deux élèves de sixième année, Kate et Sabrina, dont les scores diffèrent grandement.

Figure 1: scores généraux obtenus pour les flexions verbales $(x / 48)$

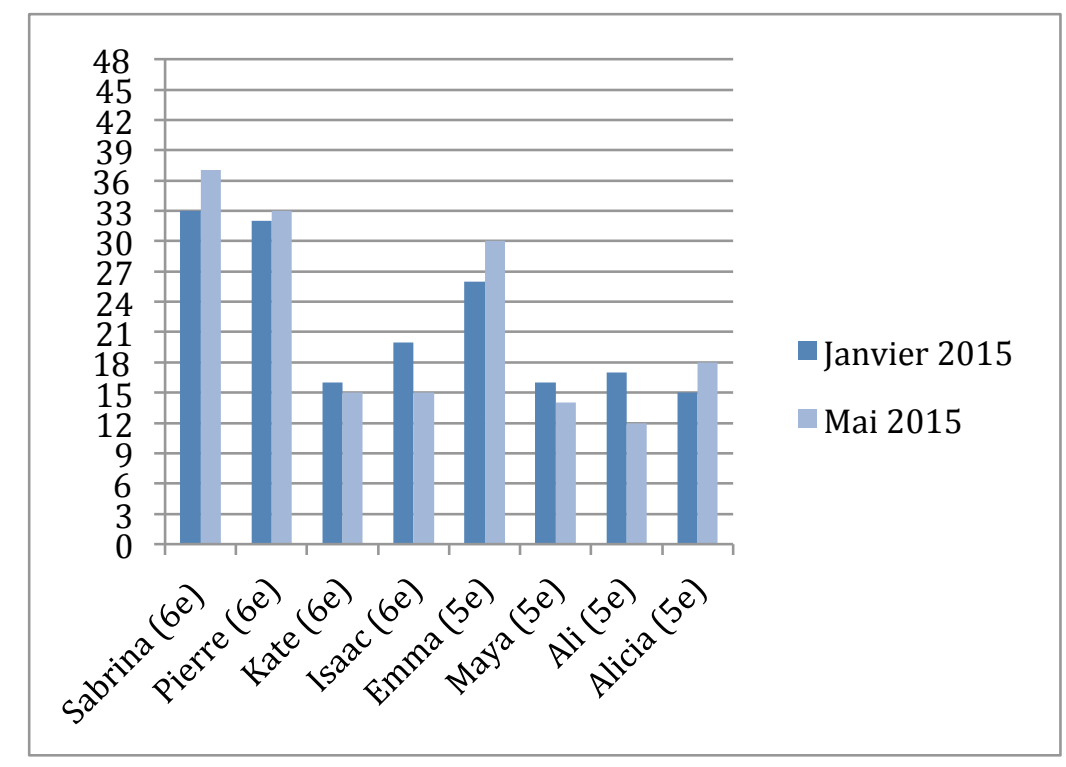

De manière générale, on remarque en premier lieu que, pour quelques élèves, la variation des scores sur la flexion d'une passation à l'autre est minime. Isaac et Ali régressent toutefois de cinq points, alors qu'Emma et Sabrina en gagnent respectivement cinq et quatre ${ }^{8}$. Pour l'assignation des marques du pluriel aux verbes, de manière générale, deux profils de scripteurs 
semblent se dessiner. D'une part, deux élèves de sixième, Sabrina et Pierre, et une de cinquième, Emma, se démarquent des autres par le respect des conventions linguistiques dont ils font montre. D’autre part, Kate, Isaac, Maya, Ali et Alicia semblent éprouver une plus grande difficulté, leurs scores, plus faibles, étant similaires les uns aux autres. On note aussi, à l'instar de Boyer (2012) en première secondaire au Québec, une disparité importante entre les résultats généraux des élèves, montrant que le marquage du pluriel verbal n'est pas encore bien intériorisé pour plusieurs de nos participants.

Figure 2: scores généraux obtenus pour les bases verbales (x/32)

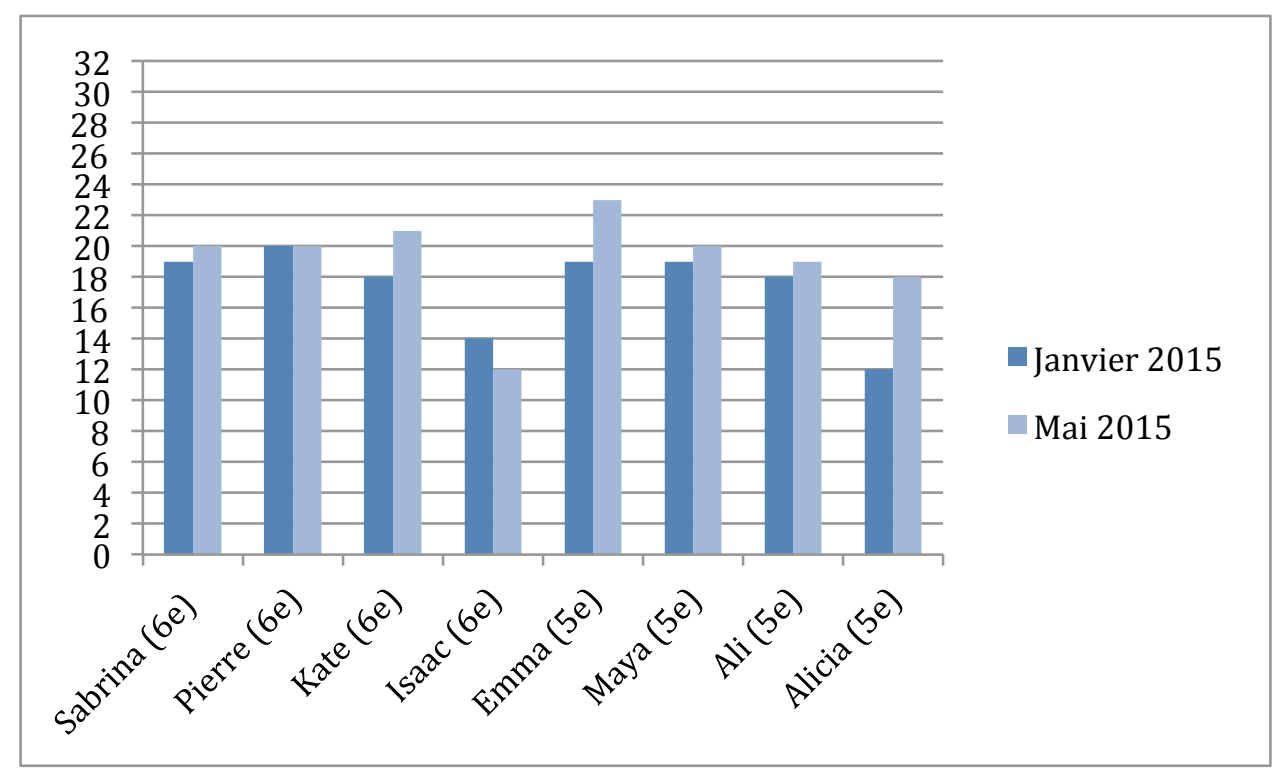

Pour l'analyse des bases verbales, il convient d'abord de mentionner que nous n'avons retenu que les 32 items pour lesquels les élèves devaient accorder des verbes à bases multiples, les verbes en -er ne convoquant pas l'altération du radical. Ainsi, comme nous le soupçonnions lorsque nous avons décidé de concevoir un cadre conceptuel composite, fondé sur des travaux en langues première et seconde, le recours à une évocation lexicale dans le choix de la base ne permet pas encore à tous coups le marquage adéquat du radical verbal pour nos participants. Cela dit, il semble que ce ne soit pas la sélection dudit radical qui nous permette de former des groupes 
de scripteurs parmi les élèves de notre étude puisque les résultats d'un élève à l'autre ne varient que peu.

Les seules différences qui méritent d'être soulevées sont, d'une part, celles dont Alicia et Emma font preuve, car leur score est à la hausse de six et de quatre points entre les deux passations. De l'autre, nous constatons qu'Isaac a régressé de deux points entre la première et la deuxième passation, lui qui en avait également perdu cinq relativement aux désinences entre les deux passations. Cette perte, qui touche donc autant le marquage lexical que le marquage morphologique des verbes, est fort surprenante, car Isaac, élève dont la famille est ougandaise, est le seul de nos participants qui se déclare uniquement francophone. D'après les dires de l'enseignante, il aurait toutefois régressé en français depuis son arrivée au Canada ${ }^{9}$. Serait-ce possible que la triple minorisation qu'il subit (Gérin-Lajoie et Jacquet, 2008) façonne son apprentissage du français écrit? Une telle hypothèse mérite selon nous d'être explorée, et nous le ferons dans le travail doctoral que nous sommes actuellement en train de terminer.

\section{Quelques résultats de Sabrina et de Kate}

Reste maintenant, à la suite d'une présentation plus générale des scores, à présenter quelques-uns des élèves et les accords qu'ils proposent, et ce, en tenant compte des environnements morphosyntaxiques que l'on retrouve dans l'épreuve. Nous retenons les profils de Kate et de Sabrina, toutes deux en sixième année, qui présentent cependant des résultats globaux fort différents. Nous rapporterons les réponses qu'elles ont offertes aux items qui adoptent deux configurations syntaxiques distinctes : les six premiers renferment comme sujet un pronom pluriel (Ils ou Elles), et les six autres présentent un sujet plus complexe, celui-ci se réalisant sous la forme d'un groupe nominal dont le noyau est singulier et qui contient un écran pluriel (p. ex., Le bébé des voisins [pleurer] très souvent).

Tableau 2: Accord des verbes de Sabrina et de Kate (sujet : pronom pluriel)

\begin{tabular}{lllll}
\hline \multicolumn{1}{c}{ Items } & \multicolumn{1}{c}{$\begin{array}{c}\text { Sabrina } \\
\text { (janvier 2015) }\end{array}$} & $\begin{array}{c}\text { Sabrina } \\
\text { (mai 2015) }\end{array}$ & $\begin{array}{c}\text { Kate } \\
\text { (janvier 2015) }\end{array}$ & $\begin{array}{c}\text { Kate } \\
(\text { mai 2015) }\end{array}$ \\
\hline Ils (porter) de très beaux vêtements aujourd'hui. & portent & portent & $*$ porte & $*$ porte \\
Elles (chercher) une solution à leur problème. & cherchent & cherchent & $*_{\text {cherche }}$ & $*$ cherche \\
Elles (être) de couleur verte. & sont & sont & $*$ est & $*$ est \\
Ils (aller) au Mexique chaque année. & $*$ allent & $*$ allent & vont & vont \\
\hline
\end{tabular}

Bellaterra Journal of Teaching \& Learning Language \& Literature. 9.3 (Aug-Sept 2016) ISSN 2013-6196 


\begin{tabular}{lllll}
\hline Ils (dire) toujours la vérité à leurs parents. & $*$ ditent & $*$ ditent & $*$ dit & $*$ dit \\
Elles (agir) comment l'enseignant l'a demandé. & $*_{\text {agient }}$ & $*_{\text {agisent }}$ & $*$ agit & $*$ agit \\
\hline
\end{tabular}

Sommairement, grâce aux verbes portent et cherchent, on remarque que Sabrina a déjà intériorisé la désinence qui indique le pluriel verbal, mais que ce n'est pas encore le cas de Kate, dont les conceptions relativement à cet objet d'apprentissage sont encore lacunaires. Pour Sabrina, la marque du pluriel - nt est d'ailleurs présente dans tous les cas, et ce, même si l'altération du radical n'a pas été opérée convenablement pour plusieurs verbes (*ditent, *agient, *agisent, ${ }^{*}$ allent $\left.^{10}\right)$. En ce qui concerne Kate, l'ensemble des bases verbales, à l'exception du verbe vont, n'a pas été modifié adéquatement. Puisqu'elle peine à mettre à l'écrit la marque du pluriel - nt, il est d'ailleurs probable que ce soit une évocation lexicale qui ait engendré l'accord du verbe vont, bien orthographié sur les plans lexical et morphologique.

Jetons maintenant un œil aux choix orthographiques de Kate et de Sabrina lorsque l'accord est complexifié par la présence d'un écran pluriel entre le verbe et son donneur d'accord au singulier.

Tableau 3: Accord des verbes de Sabrina et de Kate (sujet : groupe nominal avec écran)

\begin{tabular}{|c|c|c|c|c|}
\hline Items & $\begin{array}{l}\text { Sabrina } \\
\text { (janvier 2015) }\end{array}$ & $\begin{array}{l}\text { Sabrina } \\
\text { (mai 2015) }\end{array}$ & $\begin{array}{l}\text { Kate } \\
\text { (janvier 2015) }\end{array}$ & $\begin{array}{l}\text { Kate } \\
\text { (mai 2015) }\end{array}$ \\
\hline L'homme aux yeux verts (danser) très bien. & danse & danse & danse & *dance \\
\hline Le bébé des voisins (pleurer) très souvent. & pleure & pleure & $*$ pleur & *pleur \\
\hline $\begin{array}{l}\text { Le chat de ses amis (faire) beaucoup de bruit en } \\
\text { mangeant. }\end{array}$ & fait & fait & fait & fait \\
\hline $\begin{array}{l}\text { Le médecin de mes parents (avoir) beaucoup de } \\
\text { patients à voir aujourd'hui. }\end{array}$ & *à & a & avait $^{11}$ & a \\
\hline Le papa de tes cousins (tenir) la main de Marie. & *tenez & $*_{\text {tein }}$ & $*$ tiene & *tiene \\
\hline $\begin{array}{l}\text { Un ami de mes frères (rendre) souvent visite à } \\
\text { Pierre. }\end{array}$ & rend & *rende & rend & *rende \\
\hline
\end{tabular}

Pour ce qui est de la deuxième construction syntaxique que nous analysons dans cet article, nous observons d'emblée que ni Kate ni Sabrina ne semblent avoir subi l'effet d'attraction : en effet, aucun des verbes n'est accordé avec l'écran pluriel qui les sépare de leur donneur d'accord singulier. On peut toutefois se demander, à la lumière des réponses que Kate a produites pour les items avec un sujet se manifestant sous la forme d'un pronom pluriel, si elle n'a pas accordé les verbes avec le nom le plus proche parce qu'elle n'a pas encore intériorisé la flexion -nt. En effet, comme le soulignent Gunnarsson-Largy et Largy (2013), l'accord par 
proximité est dû à une expertise naissante, le scripteur étant en train d'automatiser le traitement de l'accord sujet-verbe. Nous avançons toutefois que, pour Kate, les connaissances relatives à la pluralité verbale et à sa marque prototypique sont encore à leurs balbutiements ; de ce fait, elle n'aurait pas encore développé ladite expertise naissante, celle qui l'inciterait à prendre en considération l'environnement syntaxique quand elle orthographie le verbe et, potentiellement, à l'accorder avec le mot qui le précède. Elle ne proposerait dès lors que la marque avec laquelle elle est familière, celle du singulier, qui s'avère être adéquate dans ces phrases. De son côté, pour éviter le piège de l'accord par proximité, Sabrina a recouru à une opération métalinguistique, car pour plusieurs des items, elle a indiqué sur sa feuille le pronom personnel qui pouvait remplacer le sujet au-dessus de ce dernier ${ }^{12}$. Elle semble donc avoir dépassé l'étape de l'expertise naissante, car elle a su reconnaitre la complexité syntaxique des items, recourir à un outil analytique pertinent et, en fonction des caractéristiques morphologiques du noyau du groupe en fonction sujet, récupérer les désinences justes.

Pour les verbes en -er, on remarque chez Sabrina que les désinences sont choisies adéquatement. Kate, quant à elle, les omet toutefois dans le verbe pleure; il est possible que cette absence de marque morphologique renvoie à une influence translinguistique de l'anglais, qui n'indique pas toujours la finitude des verbes à l'aide d'un morphogramme (p. ex., I accept, you present). On peut d'ailleurs observer que Kate a écrit le verbe danse en recourant au patron orthographique anglais en mai 2015, le $s$ étant remplacé par un $c$ dans sa réponse. Enfin, la base des verbes avoir, tenir et rendre semble encore être fragile pour les deux scriptrices, qui n'ont probablement pas développé les connaissances lexicales qui permettraient le changement de la base dans ces cas.

\section{Conclusion et pistes didactiques}

La recherche dont nous présentons ici les premiers résultats a pour objectif la description des connaissances relatives à l'accord du verbe en nombre à l'écrit chez huit élèves scolarisés dans le Sud-Ouest ontarien ${ }^{13}$, un contexte socioculturel où le français ne jouit pas d'un statut sociodémographique majoritaire. Si le devis par l'intermédiaire duquel se réalise notre étude nous

Bellaterra Journal of Teaching \& Learning Language \& Literature. 9.3 (Aug-Sept 2016)

ISSN 2013-6196 
oblige à tirer des conclusions avec circonspection, les résultats préliminaires indiquent que les connaissances de nos participants sur l'objet que nous avons ciblé ne sont pas encore stabilisées, comme on le remarque aussi chez les élèves du même âge au Québec, en milieu francophone majoritaire (Boyer, 2012; Lefrançois, 2009). Ces connaissances sont par ailleurs fort disparates, variant considérablement d'un élève à l'autre. En effet, certains élèves, à l'instar de Kate, n'ont pas encore intériorisé les marques de pluralité qui caractérisent l'accord verbal en nombre, tandis que d'autres, comme Sabrina, savent les mettre à l'écrit aux moments opportuns et utiliser la manipulation de pronominalisation pour résoudre des cas de figure plus complexes.

Nous avons également fait état de la difficulté qu'éprouvent les élèves participant à notre recherche en ce qui a trait à l'altération du radical des verbes qui sont dotés de plusieurs bases. Cette caractéristique semble généralisée chez les scripteurs de notre étude, qui accusent un niveau de connaissances qui ne fluctuent que peu d'un élève à l'autre. Nous postulons de surcroit, comme le fait Ågren $(2005 ; 2008)$ en langue seconde, que le caractère irrégulier de certaines marques lexicales associées aux verbes fréquents en français n'empêche pas le marquage flexionnel pluriel, comme l'attestent certaines des graphies proposées par Sabrina (p. ex., Ils *allent, Ils *ditent).

Bien que nous en soyons encore à nos premières analyses et que celles que nous mènerons sur les données obtenues à partir d'autres sources (productions écrites, entretiens métagraphiques) favoriseront une description plus précise des connaissances de nos participants sur l'accord du verbe en nombre à l'écrit, nous souhaitons d'ores et déjà mettre en évidence certaines considérations didactiques, qui devront probablement être nuancées ultérieurement. En milieu minoritaire, une considération de l'accord du verbe en nombre à l'écrit selon deux pans distincts, le marquage morphologique et le marquage lexical, nous semble être une avenue prometteuse pour soutenir les élèves dans leurs apprentissages du pluriel verbal. Du côté morphologique, il parait important d'offrir un enseignement explicite des manipulations syntaxiques, qui facilitent le repérage du sujet et qui permettent donc l'accord du verbe (Gauvin, 2011). Cela dit, de telles manipulations requièrent de l'élève qu'il pose un jugement de grammaticalité sur son résultat et que, par conséquent, il recoure à son bassin de connaissances 
implicites. Parce que ces dernières sont développées grâce à une exposition fréquente à la langue (Nadeau et Fisher, 2011), on peut se demander si l'ensemble des élèves en contexte minoritaire pourra utiliser ces manipulations à dessein pour résoudre les problèmes orthographiques auxquels ils font face. Il s'agit là d'une question que nous aborderons plus en détail dans notre thèse, de laquelle sont tirées les données analysées pour cet article (Thibeault, à paraitre).

Sur le plan du lexique, il nous paraitrait pertinent d'enseigner les bases des verbes selon leur fréquence d'occurrence et en fonction d'une typologie linguistique rigoureuse. Une transposition didactique des travaux du linguiste Jean Dubois (1967), en grammaire structurale, pourrait être une piste qui mériterait d'être explorée. Ce chercheur a notamment élaboré une nomenclature des verbes qui repose sur la variation de leurs radicaux, ce qui a permis l'émergence de sept catégories. Sans vouloir entrer dans le détail, soulignons qu'un traitement du verbe qui trouve son ancrage théorique dans une telle typologie offrirait probablement aux élèves une vision holistique et cohérente du système verbal, un système qui, contrairement à la croyance populaire, renferme davantage de régularités que d'exceptions. Il est également fort à parier qu'un tel dispositif didactique permette au passage de répondre aux besoins linguistiques particuliers des élèves en Ontario français et de promouvoir chez eux le développement de connaissances linguistiques qui sont en harmonie avec la langue française standard, à laquelle ils n'ont qu'un accès limité dans leur vie de tous les jours.

\section{Références}

Ågren, M. (2008). À la recherche de la morphologie silencieuse: sur le développement du pluriel en français L2 écrit (thèse de doctorat). Université de Lund, Suède.

Ågren, M. (2005). Acquisition de la morphologie silencieuse en français langue seconde à l'écrit: le cas de l'accord en nombre. Récupéré le 2 février 2013 du site: http://rudar.ruc.dk/bitstream/1800/8478/1/Artike192.pdf

Bernier, M. (2013). L'ajout d'erreurs: regard sur un épiphénomène du déficit du savoir grammatical en français au postsecondaire en milieu francophone minoritaire. Éducation francophone en milieu minoritaire, 8(1), 1-17. https://doi.org/10.21230/f39g6z

Blain, S. (2003). L'enseignement de l'écriture en milieu minoritaire canadien : problématique particulière et complémentarité des cadres théoriques en L1 et L2. Dans J.-M. Defays, J.L. Delcominette, J.-L. Dumortier et V. Louis (dir.), Langue et communication en classe de français. Convergences didactiques en langue maternelle, langue seconde et langue étrangère (p. 185-200). Cortil-Wodon, Belgique: Éditions Modulaires Européennes. 
Boyer, P. (2012). La compétence morphographique d'élèves de première secondaire. L'effet du biais d'évaluation de son efficacité personelle sur la performance (thèse de doctorat, Université du Québec à Montréal, Canada). Récupéré d'Archipel, le dépôt institutionnel numérique de l'UQAM, le 19 mars 2016: http://www.archipel.uqam.ca/5352/1/D2405.pdf

Cogis, D. (2013). Du prescrit au réel en CM2 : l'accord sujet-verbe dans le corpus Grenouille. Dans C. Gunnarsson-Largy et E. Auriac-Slusarczyk (dir.), Écriture et réécritures chez les élèves. Un seul corpus, divers genres discursifs et méthodologies d'analyse (p. 61-84). Louvain-la-Neuve, Belgique: Academia-L'Harmattan.

Dubois, J. (1967). Grammaire structurale du français : le verbe. Paris, France : Larousse.

Duquette, G. (2006). Le bilinguisme des élèves inscrits dans les écoles secondaires de langue française de l'Ontario: perceptions, valeurs et comportement langagier. Revue des sciences de l'éducation, 32(3), 665-689. https://doi.org/10.7202/016281ar

Fayol, M. et Got, C. (1991). Automatisme et contrôle dans la production écrite. L'Année psychologique, 91, 187-205. https://doi.org/10.3406/psy.1991.29453

Fleuret, C. et Armand, F. (2012). Le créole c'est presque la même affaire du français, on fait des formules...c'est déformé : Les représentations des langues par des enfants haïtiens. Revue canadienne de linguistique appliquée, 15(1), 42-59.

Gagnon, Y.-C. (2012). L'étude de cas comme méthode de recherche ( $2^{\mathrm{e}}$ éd.). Québec, Canada: PUQ.

Gauvin, I. (2011). Interactions didactiques en classe de français : enseignement/apprentissage de l'accord du verbe en première secondaire (thèse de doctorat, Université de Montréal, Canada). Récupéré de Papyrus, le dépôt institutionnel numérique de l'Université de Montréal, le 18 janvier 2016 : https://papyrus.bib.umontreal.ca/xmlui/handle/1866/5281

Gérin-Lajoie, D. (2012). L'école et le rapport à l'identité chez les jeunes des minorités de langue officielle. Récupéré le 31 juillet 2015 du site de la Fédération canadienne des enseignantes et des enseignants:

https://www.ctf-fce.ca/Publication-Library/ArticledeDianeG\%C3\%A9rinLajoie.pdf

Gérin-Lajoie, D. et Jacquet, M. (2008). Regards croisés sur l'inclusion des minorités en contexte scolaire francophone minoritaire au Canada. Éducation et francophonie, 36(1), 25-43. https://doi.org/10.7202/018088ar

Gunnarsson, C. (2012). The development of complexity, accuracy and fluency in the written production of L2 French. Dans A. Housen, F. Kuiken et I. Vedder (dir.), Dimensions of L2 performance and proficiency. Complexity, accuracy and fluency in SLA (p. 247-276). Amsterdam, Pays-Bas : John Benjamins Publishing Company.

Gunnarsson-Largy, C. et Largy, P. (2013). Le développement des automatismes en production écrite du CM2 à la $4^{\mathfrak{e}}$ collège à travers l'accord de proximité et les récupérations d'instances. Dans C. Gunnarsson-Largy et E. Auriac-Slusarczyk (dir.), Écriture et réécritures chez les élèves. Un seul corpus, divers genres discursifs et méthodologies d'analyse (p. 129-152). Louvain-la-Neuve, Belgique: Acadmia-L'Harmattan.

Jaffré, J.-P. (1995). Compétence orthographique et acquisition. Dans D. Ducard, R. Honvault et J.-P. Jaffré (dir.), L'orthographe en trois dimensions (p. 94-158). Paris, France: Nathan.

Bellaterra Journal of Teaching \& Learning Language \& Literature. 9.3 (Aug-Sept 2016) ISSN 2013-6196 
Laflamme, S. et Reguigui, A. (2003). Homogénéité et distinction. Sudbury, Canada: Prise de parole.

Lefrançois, P. (2009). Évolution de la conception du pluriel des noms, des adjectifs et des verbes chez les élèves du primaire. Repères, 39, 187-206. https://doi.org/10.4000/reperes.846

Ministère de l'Éducation de l'Ontario (2011). L'éducation en langue française en Ontario. Récupéré le 27 septembre 2015 du site du ministère : http://www.edu.gov.on.ca/fre/amenagement/

Ministère de l'Éducation de l'Ontario (2006). Le curriculum de l'Ontario de la $1^{\text {re }}$ à la $8^{e}$ année. Français. Récupéré le 14 septembre 2014 du site du ministère: http://www.edu.gov.on.ca/fre/curriculum/elementary/language18currb.pdf

Ministère de l'Éducation du Québec (2001). Programme de formation de l'école québécoise. Éducation préscolaire, enseignement primaire. Québec, Canada: Gouvernement du Québec.

Nadeau, M. et Fisher, C. (2011). Les connaissances implicites et explicites en grammaire: quelles importances pour l'enseignement? Quelles conséquences? Bellaterra Journal of Teaching \& Learning Language \& Literature, 4(4), 1-31. Récupéré le 15 septembre 2016 du: http://revistes.uab.cat/jt13/article/view/446/496

Ouellet, C., Wagner, A. et Boily, É. (2013). Les accords grammaticaux dans le groupe du nom. Comment des élèves de classes ordinaires de la fin du primaire, du début du secondaire et des élèves présentant des difficultés d'apprentissage s'y prennent-ils? Dans D. Daigle, I. Montésinos-Gelet et A. Plisson (dir.), Orthographes et populations exceptionnelles. Perspectives didactiques (p. 37-56). Québec, Canada: Presses de l'Université du Québec.

Pellat, J.-C. (2009). Quelle grammaire enseigner? Paris, France: Hatier.

Thibeault, J. (à paraitre). L'apprentissage du français écrit en milieu minoritaire : le cas d'élèves de la fin de l'ordre élémentaire scolarisés dans le sud-ouest de l'Ontario et de leur accord du verbe en nombre (thèse de doctorat, Université d'Ottawa, Canada).

Totereau, C. Brissaud, C., Reilhac, C. et Bosse, M. L. (2013). L'orthographe grammaticale au collège: une approche sociodifférenciée. A.N.A.E., 123, 164-171.

Totereau, C. Thevenin, M.-G. et Fayol, M. (1997). Acquisition de la morphologie du nombre à l'écrit en français. Dans L. Rieben, M. Fayol et C. A. Perfetti (dir.), Des orthographes et leur acquisition (p. 147-166). Lausanne, Suisse: Delachaux et Niestlé.

Information sur l'auteur: Joël Thibeault est candidat au doctorat à l'université d'Ottawa et professeur à l'université de Regina, et son travail porte sur l'enseignement et l'apprentissage de la grammaire chez les élèves scolarisés au sein d'écoles de langue française en milieu minoritaire. Il s'implique également dans les formations initiale et continue des enseignants qui sont appelés à enseigner le français à des élèves qui n'ont pas nécessairement un accès constant à cette langue à l'extérieur de l'enceinte scolaire.

Email: joel.thibeault@uregina.ca

Information sur l'auteure: Carole Fleuret est professeure agrégée à la Faculté d'éducation de l'Université d'Ottawa. Ses intérêts de recherche portent sur l'appropriation de l'écrit, entre autres, sur le développement de la compétence orthographique en langue seconde et sur l'étude des composantes cognitives, socio-culturelles et affectives qui soutiennent ou freinent cette appropriation. Ses travaux s'intéressent aux populations exceptionnelles en contexte multiethnique et plurilingue.

Bellaterra Journal of Teaching \& Learning Language \& Literature. 9.3 (Aug-Sept 2016) ISSN 2013-6196 
1 Selon Statistique Canada (2011), 68,3\% des couples ontariens qui ont des enfants et dont l'un des membres est francophone vivent en relation exogame. Dans le Sud-Ouest de la province, milieu de notre étude, ce pourcentage grimpe à $87,5 \%$. Puisque plusieurs parents dont les enfants ont accès à l'école de langue française décident de scolariser leurs enfants dans une école de la majorité anglophone, certains conseils scolaires francophones de l'Ontario acceptent maintenant des élèves dont les parents ne sont pas des ayants droit. Ces conseils sont dotés de comités d'admission, qui permettent (ou non) aux élèves de foyers non francophones de fréquenter les établissements scolaires de langue française.

${ }_{2}^{2}$ Il convient de rappeler que, pour ce qui est du verbe, la distinction singulier-pluriel ne peut s'opérer qu'à la troisième personne. Comme le mentionne Pellat (2009), la première personne du pluriel ne constitue pas toujours le pluriel de la première personne du singulier, de même que la deuxième personne du pluriel ne renvoie pas toujours à la deuxième personne du singulier. Comme l'ensemble des chercheurs qui s'intéressent au nombre verbal, nous nous concentrerons donc sur les troisièmes personnes du singulier et du pluriel.

${ }^{3}$ Nous définirons la supplétion comme étant un phénomène d'irrégularité qui touche certains verbes qui ne sont pas construits par la simple modification d'un radical unique. La forme verbale supplétive ne sera donc pas une forme prédictible à partir de la base, ici l'infinitif.

${ }^{4}$ Dans le cadre de cette activité, on présente à l'écrit un ensemble de phrases à l'élève, et la forme infinitive des verbes dans les phrases apparait entre parenthèses. L'élève est alors appelé à accorder les verbes au présent de l'indicatif.

${ }^{5}$ Pour des raisons déontologiques, chaque élève s'est vu attribuer un pseudonyme.

${ }^{6}$ Une didacticienne de la grammaire et une didacticienne des langues secondes nous ont offert une évaluation rigoureuse de notre instrument. Qu'elles trouvent ici notre plus sincère reconnaissance.

${ }^{7}$ Le caractère qualitatif de nos analyses ne nous permet pas de valider notre instrument à l'aide des procédures statistiques qui sont souvent utilisées lors d'études adoptant des méthodes quantitatives. Il s'agit là d'une limite de notre recherche.

${ }^{8}$ Ces variations sont difficilement explicables pour l'instant. Les commentaires métagraphiques nous permettront probablement de voir une différence entre les raisonnements qui ont mené aux graphies d'une passation à l'autre.

${ }^{9}$ Elle nous explique : « Il est arrivé avec un français très bon. Mais depuis qu'il est ici, il parle seulement en anglais. J'essaye de lui dire de parler français, mais ses amis lui parlent en anglais. Pis ça se voit dans ses résultats ».

${ }^{10}$ Il est possible que, en accordant le verbe aller, Sabrina n'ait pas su se distancier de la forme infinitive, qui apparaissait entre parenthèses, et que sa présence ait influencé les réponses qu'elle a offertes. Si, contrairement à la dictée, l'activité de complètement offre l'avantage de pouvoir mesurer les connaissances quant à l'altération du radical, elle peut également offrir de fausses pistes à l'élève quand les verbes à accorder, comme aller, revêtent une forme supplétive lorsqu'ils sont fléchis.

${ }^{11}$ Lors de l'entretien métagraphique, nous avons demandé à Kate à quel temps était ce verbe pour elle. Elle nous a répondu qu'il était au présent.

${ }^{12}$ En grammaire nouvelle, la pronominalisation est l'une des manipulations syntaxiques décisives qui permet l'identification du sujet et, par ricochet, l'accord du verbe (Gauvin, 2011).

${ }^{13}$ La mention du contexte géographique est importante, car les connaissances linguistiques des élèves peuvent être fluctuantes d'un milieu minoritaire à un autre. 
Annexe A: Tableau montrant la construction des items faisant partie de l'activité de complètement

\begin{tabular}{|c|c|c|c|c|}
\hline Constructions syntaxiques retenues & Exemples d'item & $\begin{array}{l}\text { Verbes en } \\
-e r \\
\text { (nombre } \\
\text { d'items) }\end{array}$ & $\begin{array}{l}\text { Verbes être, } \\
\text { avoir, faire, } \\
\text { aller (nombre } \\
\text { d'items) }\end{array}$ & $\begin{array}{l}\text { Autres } \\
\text { verbes } \\
\text { (nombre } \\
\text { d'items) }\end{array}$ \\
\hline $\begin{array}{c}\text { Pronom singulier en fonction } \\
\text { sujet }\end{array}$ & $\begin{array}{c}\text { Il (trouver) beaucoup } \\
\text { d'informations sur les sites } \\
\text { Internet. }\end{array}$ & 2 & 2 & 2 \\
\hline $\begin{array}{l}\text { Groupe nominal singulier (court) } \\
\text { en fonction sujet }\end{array}$ & $\begin{array}{l}\text { Le camion rouge (passer) près } \\
\text { de notre maison tous les matins. }\end{array}$ & 2 & 2 & 2 \\
\hline Pronom pluriel en fonction sujet & $\begin{array}{l}\text { Ils (dire) toujours la vérité à } \\
\text { leurs parents. }\end{array}$ & 2 & 2 & 2 \\
\hline $\begin{array}{l}\text { Groupe nominal pluriel (court) } \\
\text { en fonction sujet }\end{array}$ & $\begin{array}{l}\text { Samuel et Paolo (prendre) les } \\
\text { tomates sur la table. }\end{array}$ & 2 & 2 & 2 \\
\hline $\begin{array}{l}\text { Long groupe nominal sujet au } \\
\text { pluriel (énumération) }\end{array}$ & $\begin{array}{l}\text { Ma sœur, mes deux frères, mes } \\
\text { parents, ma tante et mes voisins } \\
\text { (rester) dehors pendant les feux } \\
\text { d'artifice. }\end{array}$ & 2 & 2 & 2 \\
\hline $\begin{array}{c}\text { Sujet se réalisant sous la forme } \\
\text { d'un groupe nominal dans lequel } \\
\text { on retrouve des noms dotés de } \\
\text { nombres différents }\end{array}$ & $\begin{array}{l}\text { Le chat de ses amis (faire) } \\
\text { toujours du bruit en mangeant. }\end{array}$ & 2 & 2 & 2 \\
\hline $\begin{array}{c}\text { Sujet singulier évoquant la } \\
\text { pluralité }\end{array}$ & $\begin{array}{l}\text { La population (aller) dans les } \\
\text { écoles pour voter. }\end{array}$ & 2 & 2 & 2 \\
\hline $\begin{array}{l}\text { Ordre canonique sujet-prédicat- } \\
\text { complément non respecté }\end{array}$ & $\begin{array}{c}\text { Dans le casier (être) les } \\
\text { manteaux. }\end{array}$ & 1 & 1 & 1 \\
\hline $\begin{array}{c}\text { Sujet se réalisant sous la forme } \\
\text { d'un groupe nominal dans lequel } \\
\text { il y a une phrase subordonnée } \\
\text { relative }\end{array}$ & $\begin{array}{l}\text { Le mur que nous décorons en } \\
\text { chantant de belles chansons } \\
\text { (être) très grand. }\end{array}$ & 1 & 1 & 1 \\
\hline
\end{tabular}

Pour citer cet article:

Thibeault, J., \& Fleuret, C. (2016). L'accord du verbe en nombre à l'écrit chez des élèves de la fin de l'élémentaire en Ontario français: Une étude de cas. Bellaterra Journal of Teaching \& Learning Language \& Literature, 9 (3): $32-$ 49. DOI: http://dx.doi.org/10.5565/rev/jt13.651

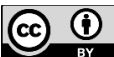

